\title{
Slow Group Velocity and Cherenkov Radiation
}

\author{
I. Carusotto, ${ }^{1,2, *}$ M. Artoni, ${ }^{3}$ G. C. La Rocca, ${ }^{1,2}$ and F. Bassani ${ }^{2}$ \\ ${ }^{1}$ Dipartimento di Fisica “E. R. Caianiello,” Università di Salerno, via S. Allende, I-84081 Baronissi (Sa), Italy \\ ${ }^{2}$ INFM, Scuola Normale Superiore, Piazza dei Cavalieri 7, 56126 Pisa, Italy \\ ${ }^{3}$ INFM, European Laboratory for non-Linear Spectroscopy, Largo E. Fermi 2, 50125 Florence, Italy
}

(Received 12 March 2001; published 18 July 2001)

\begin{abstract}
We study theoretically the effect of ultraslow group velocities on the emission of Vavilov-Cherenkov radiation in a coherently driven medium. We show that in this case the aperture of the group cone on which the intensity of the radiation peaks is much smaller than that of the usual wave cone associated with the Cherenkov coherence condition. As a specific example, we consider a coherently driven ultracold atomic gas where such singular behavior may be observed.
\end{abstract}

The recent observation of ultraslow group velocities in coherently driven media [1-3] has opened the way towards new regimes of light propagation [4] and nonlinear optics at very weak intensities [5]. In this Letter, we investigate the effect of ultraslow group velocities on the Cherenkov radiation emitted by a charged particle uniformly moving with a velocity larger than the phase velocity of light. In the case of isotropic and nondispersive media, the surface on which the intensity peaks coincides with the well-known wave cone orthogonal to the wave vector of the emitted light and the aperture of which is determined by the usual Cherenkov coherence condition. In the case of highly dispersive media, as we show here, the intensity is instead peaked on the surface of a group cone [6] much narrower than the wave cone and orthogonal neither to the phase nor to the group velocity of the emitted light. First, we develop a general theory for Cherenkov emission in arbitrary dispersive and nonisotropic media from which we obtain analytical expressions for the electric field intensity profile and, in particular, for the group cone aperture. Second, for illustrative purposes, we investigate the optical properties of a coherently driven ultracold atomic cloud of ${ }^{23} \mathrm{Na}$ atoms which appears to be amenable to the observation of such singular behavior. A clear distinction between wave cone and group cone is expected to clarify the role of the group velocity in the process of Cherenkov emission.

Consider a charged pointlike particle moving with constant velocity $\mathbf{w}=w \hat{\mathbf{z}}=c \beta \hat{\mathbf{z}}$ through a nonabsorptive and homogeneous medium characterized by an anisotropic and dispersive Hermitian dielectric tensor $\hat{\epsilon}(\omega)=\epsilon_{i, j}(\omega)$. The effects of weak absorption will be considered later for the case of interest. Using Maxwell equations in Fourier space, we write the $(\mathbf{k}, \omega)$ component of the radiated electromagnetic field $\mathbf{E}(\mathbf{k}, \omega)$ in terms of the corresponding Fourier components of the current density $\mathbf{J}(\mathbf{k}, \omega)=$ $2 \pi q \delta(\omega-\mathbf{k} \cdot \mathbf{w}) \mathbf{w}$ as

$$
\mathbf{E}(\mathbf{k}, \omega)=\frac{4 \pi i \omega}{c^{2}}\left(k^{2} \hat{\mathcal{P}}^{\mathbf{k}}-\frac{\omega^{2}}{c^{2}} \hat{\boldsymbol{\epsilon}}(\omega)\right)^{-1} \mathbf{J}(\mathbf{k}, \omega),
$$

where $\hat{\mathcal{P}}_{i, j}^{\mathbf{k}}=\delta_{i, j}-\frac{k_{i} k_{j}}{k^{2}}$ is the projection operator on the subspace orthogonal to $\mathbf{k}$ and $k^{2}=\sum_{i} k_{i} k_{i}$ is the square modulus of $\mathbf{k}$.

The poles of (1) determine the propagating modes of the electromagnetic field through the well-known Fresnel equation [7]

$$
\left(k^{2} \hat{\mathcal{P}^{\mathbf{k}}}-\frac{\omega_{\alpha}^{2}}{c^{2}} \hat{\boldsymbol{\epsilon}}\left(\omega_{\alpha}\right)\right) \mathbf{e}^{(\alpha)}=0 .
$$

For each wave vector $\mathbf{k}$, the different modes are characterized by the frequency $\omega_{\alpha}$ and the polarization unit vector $\mathbf{e}^{(\alpha)}$ normalized as $\left\langle\mathbf{e}^{(\alpha)} \mid \mathbf{e}^{(\alpha)}\right\rangle=\sum_{i} \mathbf{e}_{i}^{(\alpha) *} \mathbf{e}_{i}^{(\alpha)}=1$. We will not account here for longitudinal modes, assuming $\omega^{2} \neq 0$ and $\operatorname{det}[\hat{\epsilon}(\omega)] \neq 0$ for all values of $\omega$. For each mode, the group velocity can be shown to be [8]

$$
\begin{aligned}
\mathbf{v}_{g}^{(\alpha)} & =\nabla_{\mathbf{k}} \omega_{\alpha} \\
& =c^{2} \frac{2 \mathbf{k}-\mathbf{e}^{(\alpha)}\left\langle\mathbf{e}^{(\alpha)} \mid \mathbf{k}\right\rangle-\mathbf{e}^{(\alpha) *}\left\langle\mathbf{k} \mid \mathbf{e}^{(\alpha)}\right\rangle}{\left\langle\mathbf{e}^{(\alpha)}\left|\frac{\partial}{\partial \omega}\left[\omega^{2} \hat{\boldsymbol{\epsilon}}(\omega)\right]\right| \mathbf{e}^{(\alpha)}\right\rangle} .
\end{aligned}
$$

We shall also restrict our attention to the simple case of a medium with rotational symmetry around the direction of the charge velocity; in this case, the revolution symmetry of the dispersion surface $\omega_{\alpha}(\mathbf{k})=\omega$ guarantees the parallelism of the components of the group velocity and the wave vector perpendicular to the $\hat{\mathbf{z}}$ axis.

The electric field at a position $\mathbf{x}=\left(\mathbf{x}_{\perp}, z\right)=\left(x_{\perp} \hat{\mathbf{u}}_{\perp}, z\right)$ can be obtained from the inverse Fourier transform of (1). At sufficiently large distances from the charge trajectory, only the poles of (1) effectively contribute to the Fourier transform. In fact, the electric field at these distances is given by the resonant excitation of propagating modes, while the nonresonant contribution from all other modes decays in space with a faster power law [9]. Within such an approximation we can write, for the $i$ th component,

$$
\begin{aligned}
E_{i}\left(\mathbf{x}_{\perp}, z, t\right)= & \frac{2 i q}{c^{2}} \int_{0}^{\infty} d \omega \sum_{\alpha=1,2} \frac{k_{\perp}^{(\alpha)} \omega\left\langle\mathbf{e}^{(\alpha)} \mid \hat{\mathbf{w}}\right\rangle}{\mu_{\alpha}} \\
& \times \sqrt{\frac{i}{2 \pi k_{\perp}^{(\alpha)} x_{\perp}}} e^{i k_{\perp}^{(\alpha)} x_{\perp}} e^{i(\omega / w)(z-w t)} \mathbf{e}_{i}^{(\alpha)} .
\end{aligned}
$$


Here, $\hat{\mathbf{w}}=\mathbf{w} / w$ is the direction of the charge velocity $\mathbf{w}$ and $\mu_{\alpha}=\left\langle\mathbf{e}^{(\alpha)}\left|\frac{\partial}{\partial k_{\perp}}\left(k^{2} \hat{\mathcal{P}}^{\mathbf{k}}\right)\right| \mathbf{e}^{(\alpha)}\right\rangle$ is a weight factor. In obtaining the expression (4) the angular integration in $\mathbf{k}_{\perp}$ has already been performed in the large $k_{\perp} x_{\perp}$ limit so that, for each position $\mathbf{x}_{\perp}$, only the field components with $\mathbf{k}_{\perp}$ parallel to $\mathbf{x}_{\perp}$ have a stationary phase and therefore give a nonvanishing contribution to the integral.

Depending on the velocity of the charged particle, for each direction $\hat{\mathbf{u}}_{\perp}$ and frequency $\omega$, at most two distinct poles at $k_{\perp}^{(\alpha)}(\alpha=1,2)$ contribute to the integral, which correspond to propagating modes with wave vector $\mathbf{k}^{(\alpha)}=$ $\left(k_{\perp}^{(\alpha)} \hat{\mathbf{u}}_{\perp}, \omega / w\right)$ and polarization $\mathbf{e}^{(\alpha)}$. As shown in Fig. 1, at a given frequency $\omega$ the Cherenkov light is emitted into the mode $\alpha$ only if the $k_{z}=\omega / c \beta$ plane has a nonvanishing intersection with the dispersion surface $\omega_{\alpha}(\mathbf{k})=\omega$ of the mode. In the case of a medium with rotational symmetry, this condition is satisfied if the particle velocity exceeds the phase velocity of mode $\alpha$ for $\mathbf{k}$ parallel to $\mathbf{w}$, i.e.,

$$
\beta^{2}>\left.\frac{\omega^{2}}{c^{2} k^{(\alpha) 2}}\right|_{\mathbf{k} \| \mathbf{w}}=\frac{1}{\epsilon_{\perp}^{(\alpha)}(\omega)}
$$

and the intersection is the $k_{\perp}=k_{\perp}^{(\alpha)}$ circle. Here $\epsilon_{\perp}^{(\alpha)}(\omega)$ are the eigenvalues of the dielectric constant in the plane perpendicular to the $\hat{\mathbf{z}}$ axis. For an isotropic medium, the condition (5) reduces to the usual Cherenkov condition $\beta^{2} \epsilon(\omega)>1[10]$.

The theory developed up to now has considered the case of a nonabsorptive medium for which the poles $k_{\perp}^{(\alpha)}$ are real; the effect of a weak absorption consists of the introduction of a small and positive imaginary part $\operatorname{Im}\left[k_{\perp}^{(\alpha)}\right]$ in the argument of the exponential in (4) without modifying the pole structure of the integral. The resulting damping factor $e^{-\operatorname{Im}\left[k_{\perp}^{(\alpha)}\right] x_{\perp}}$ accounts for the absorption of the emitted radiation during propagation.

We now consider a medium with a narrow transparency window centered at $\bar{\omega}$; for a single polarization state $\alpha=1$, the absorption factor in the neighborhood of $\bar{\omega}$ can be approximated by $\operatorname{Im}\left[k_{\perp}^{(1)}\right] \simeq \frac{\eta}{2}(\omega-\bar{\omega})^{2}$ with $\eta=$

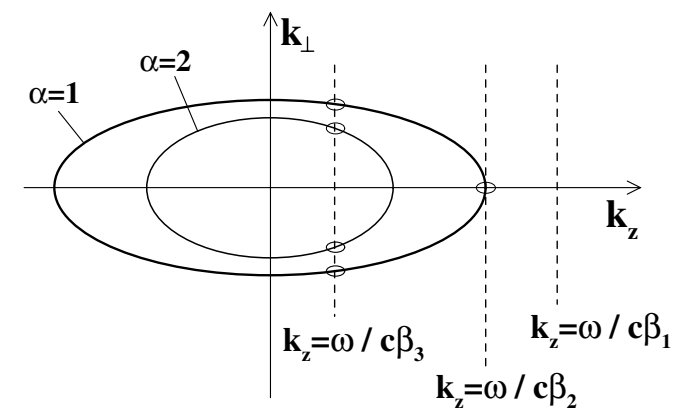

FIG. 1. Schematic plot of the longitudinal cross section of the dispersion surfaces for the two propagating $\alpha=1,2$ modes at a given $\omega$. The vertical lines are the cross sections of the $k_{z}=$ $\omega / \beta c$ planes. For $\beta=\beta_{1}$, no Cherenkov radiation occurs at $\omega ; \beta=\beta_{2}$ is the threshold velocity for the $\alpha=1$ mode; for $\beta=\beta_{3}$, both modes are excited. $\partial^{2} \operatorname{Im}\left[k_{\perp}^{(1)}\right] / \partial \omega^{2}$. Inserting this expression into (4), we finally obtain an explicit expression for the electric field intensity profile,

$$
\begin{aligned}
|\mathbf{E}(\mathbf{x}, t)|^{2}= & \frac{A q^{2}}{x_{\perp}^{2}} \exp \left[-\frac{1}{\eta x_{\perp}}\left(\frac{z}{w}+\frac{x_{\perp}}{v_{r}}-t\right)^{2}\right] \\
& \times\left|\left\langle\mathbf{e}^{(1)} \mid \hat{\mathbf{w}}\right\rangle\right|^{2},
\end{aligned}
$$

where $\mathbf{e}^{(1)}$ is the polarization vector of the mode at frequency $\bar{\omega}$ and $A=4 k_{\perp}^{(1)} \bar{\omega}^{2} / c^{4} \mu_{1}^{2} \eta$. The radial velocity $v_{r}$ is defined according to

$$
v_{r}^{-1}=\left[\left.\frac{\partial k_{\perp}}{\partial \omega}\right|_{k_{z}=\bar{\omega} / w}\right]=\frac{w-v_{g}^{\|}}{w v_{g}^{\perp}},
$$

where $v_{g}^{\perp}$ and $v_{g}^{\|}$are, respectively, the perpendicular and parallel components of the group velocity $\mathbf{v}_{g}^{(1)}$ with respect to the direction $\hat{\mathbf{w}}=\mathbf{w} / w$ of the charge velocity. As can be observed in Fig. 2, sufficiently far from the charge, i.e., at points $x_{\perp} \gg \xi=\eta v_{r}^{2}$, the electric field intensity (6) is peaked on the group cone described by

$$
\frac{x_{\perp}}{v_{r}}+\frac{z}{w}=t
$$

whose aperture $\theta$ is given by

$$
\tan \theta=v_{r} / w .
$$

In general, $\theta$ is different from the aperture $\phi$ of the wave cone orthogonal to the wave vectors of the emitted radiation, which is instead given by $\tan \phi=\bar{\omega} / w k_{\perp}^{(1)}$. A simple physical interpretation of the group cone can be put forward in terms of group velocity [6] considering that, for each direction around the charge velocity, the burst of Cherenkov light is emitted into a group of modes centered at $\mathbf{k}^{(1)}$ while the peak of the pulse moves in space with a velocity equal to the group velocity $\mathbf{v}_{g}^{(1)}$ experiencing an almost negligible absorption. The cone defined by this geometrical construction (Fig. 3) can be proven in all cases to be equivalent to the group cone defined by (8) and to coincide, for the case of an isotropic medium, with the group cone introduced by Frank [6]. Notice that this cone turns out to be in general orthogonal neither to the group



FIG. 2. Intensity contour plot of the longitudinal cross section of the group cone at $t=0$ for $v_{g}^{\perp} / w=0.01$. 
velocity nor to the wave vector. In the case of isotropic and nondispersive media, in which $\mathbf{v}_{g}=\mathbf{v}_{p h}=\frac{c}{n} \hat{\mathbf{k}}, n$ being the refractive index, the group and wave cones coincide and have an aperture $\phi$ defined by the usual Cherenkov condition $\beta n \sin \phi=1$.

The weak dispersion of common dielectrics makes the difference between the group and wave cones very small and has prevented up to now its experimental observation [6]. When the group velocity is much smaller than the phase velocity, the group cone is expected to be well separated from the wave cone (Fig. 3) and to have an extremely narrow shape, $\theta \ll 1$. Following the recent observations of ultraslow light in coherently driven hot [1,2] and cold [3] atomic gases, such media appear as promising candidates for the experimental characterization of the role of group velocity in Cherenkov radiation.

Consider a cloud of ultracold atoms [11] in a three-level $\Lambda$-type configuration and take, as a specific example, the case of ${ }^{23} \mathrm{Na}$ atoms (Fig. 4) magnetically trapped in the $M_{F}=-1$ sublevel of the $F_{g}=1$ ground state. Let $\omega_{g}$ be the frequency of such a state. The other hyperfine component of the $S_{1 / 2}$ ground state is a metastable state with $F_{m}=2$ approximately $1.8 \mathrm{GHz}$ blue-detuned with respect to the ground state. A weak coherent field polarized along the trap magnetic field drives the optical transition from the metastable state to the $F_{e}=2$ hyperfine component of the $P_{1 / 2}$ excited state. Its Rabi frequency $\Omega$ is smaller than the excited state linewidth $\gamma_{e} \simeq 2 \pi \times 10 \mathrm{MHz}$, while its frequency is taken to be exactly on resonance with the transition between the $M_{F}=0$ Zeeman components $\omega_{d r}=\omega_{e}\left[M_{F}=0\right]-\omega_{m}\left[M_{F}=0\right]$. Assuming the charge velocity to be parallel to the trap magnetic field, the rotational symmetry of the system around the $\hat{\mathbf{z}}$ axis implies [13] the following decomposition of the dielectric tensor: $\quad \hat{\boldsymbol{\epsilon}}(\omega)=\epsilon_{z}(\omega)|\hat{\mathbf{z}}\rangle\left\langle\hat{\mathbf{z}}\left|+\epsilon_{+}(\omega)\right| \hat{\sigma}_{+}\right\rangle\left\langle\hat{\sigma}_{+}\right|+$ $\epsilon_{-}(\omega)\left|\hat{\sigma}_{-}\right\rangle\left\langle\hat{\sigma}_{-}\right|$. For each frequency $\omega$ and direction $\hat{\mathbf{k}}$, the two propagating modes defined by (2) are generally nondegenerate, except at high symmetry points, and have elliptical polarizations; from the point of view of the spatial symmetry of the optical constants, the polarized atomic cloud is in fact not only uniaxial but also optically active. Since the linewidth $\gamma_{m}$ of the metastable $m$ state is orders of magnitude smaller [3] than that of the excited $e$

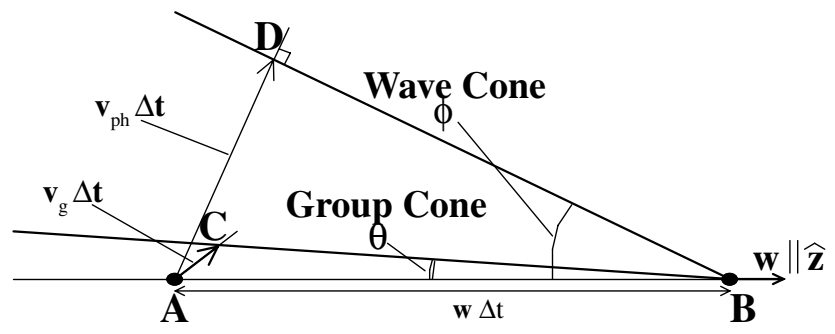

FIG. 3. Geometrical construction for the group cone. During the time $\Delta t$, the charge moves from $A$ to $B$ with $\overrightarrow{A B}=\mathbf{w} \Delta t$, while the radiation emitted in $A$ propagates from $A$ to $C$ with $\overrightarrow{A C}=\mathbf{v}_{g} \Delta t$. As discussed in the text, the straight line joining $B$ and $C$ is a generatrix of the group cone. state $\gamma_{e}$, electromagnetically induced transparency (EIT) occurs on the $\hat{\sigma}_{+}$polarization in a narrow frequency window of linewidth $\Gamma=\Omega^{2} / \gamma_{e} \ll \gamma_{e}$ around $\omega_{+}=$ $\omega_{e}\left[M_{F}=0\right]-\omega_{g}[12]$, where absorption is quenched and dispersion enhanced so as to give slow light propagation. In the same frequency window $\omega \simeq \omega_{+}$, the transitions from the ground state to the $M_{F}=-2,-1$ sublevels of the excited state are sufficiently off resonance $\left(\Delta \omega_{z,-}=\right.$ $\left.\omega_{e}\left[M_{F}=-1,-2\right]-\omega_{e}\left[M_{F}=0\right] \gg \gamma_{e}\right)$ so as to give a positive and relatively frequency-flat background contribution to the $\hat{\sigma}_{-}$and $\hat{\mathbf{z}}$ components of the dielectric tensor,

$$
\begin{gathered}
\epsilon_{+}=1+\frac{4 \pi f_{+}}{\omega_{+}-i \gamma_{e}-\omega-\frac{|\Omega|^{2}}{\omega_{+}-i \gamma_{m}-\omega}}, \\
\epsilon_{z,-}=1+4 \pi \chi_{z,-}^{\infty}=1+\frac{4 \pi f_{z,-}}{\Delta \omega_{z,-}} .
\end{gathered}
$$

The oscillator strengths $f_{ \pm, z}$ are proportional to the atomic density times the square of the dipole moment of the optical transition and differ from each other depending on the relevant Clebsch-Gordon coefficients. The detunings $\Delta \omega_{z,-}$ follow from the Zeeman splitting of the atomic levels, which implies that the background refraction can be experimentally controlled by tuning the magnetic field; with ${ }^{23} \mathrm{Na}$ atoms, a splitting $\Delta \omega_{-}$of about $2 \pi \times 40 \mathrm{MHz}$ occurs in a reasonable magnetic field of the order of $80 \mathrm{G}$. Given the different magnetic moments of metastable and excited states, the weak dressing field cannot effectively dress the optical transitions other than the resonant one between the $M_{F}=0$ sublevels.

The threshold velocity (5) for Cherenkov emission at $\omega_{+}$is determined by the background refractive index (11). If the velocity is larger than the threshold value, light is radiated into a mode with nonvanishing $k_{\perp}^{(1)}$; in the presence of EIT, such a radiation will propagate with ultraslow group velocity without being absorbed. The magnitude of the group velocity (3) is mainly determined by the dispersive properties of EIT while its direction depends on the background refractive index only. Group velocities as low as $17 \mathrm{~m} / \mathrm{sec}$ have been reported in an ultracold sodium gas [3] and this means that the group cone would

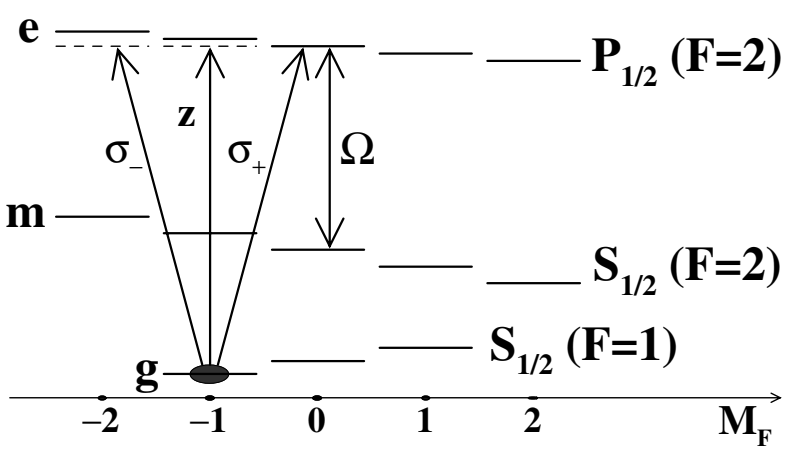

FIG. 4. Scheme of the ${ }^{23} \mathrm{Na}$ atomic levels involved in the optical process under examination. 
have an extremely narrow shape, practically a cylindrical one $(\theta \ll 1)$. From the experimentally determined parameters of this system [3], the characteristic length $\xi=$ $\eta v_{r}^{2} \simeq \eta v_{g}^{\perp 2}$ turns out to be of the order of $10 \mu \mathrm{m}$ and the background susceptibility $4 \pi \chi_{z,-}^{\infty}$ is of the order of $10^{-2}$, i.e., much larger than that usually found in gas Cherenkov counters [10].

The theory we have developed is based on the assumption of a homogeneous medium. This approximation is reasonable provided the overall size of the atomic cloud is much larger than the wavelength of the Cherenkov light whose detection should be performed within the cloud itself to avoid reflection effects at the edges of the cloud. In analogy with what has been done in the case of electrooptic materials [14], a picture of the group cone can be taken, exploiting the very large cross section for resonant two-photon absorption processes in EIT media [15]: the absorption coefficient experienced by a laser field on resonance with another optical transition starting from the $M_{F}=0$ sublevel of the metastable $m$ state is in fact proportional to the local intensity of the Cherenkov radiation at $\omega_{+}$which forms the narrow group cone [5]. Since the interaction of the charge with the atoms of the cloud results not only in Cherenkov emission but also in other heating and ionization processes [7], it is necessary to reduce the importance of such short-range processes by making the charge travel in a region of space free from atoms. For the case of an atomic cloud, this can be achieved, e.g., by using the repulsive potential of a blue-detuned laser so as to create a sort of "tunnel" through the cloud; a small cylindrical hole with a radius of the order of the wavelength does reduce the yield of the Cherenkov radiation, but does not affect the qualitative features of the pulse propagating in the surrounding medium [16]. Unfortunately, the intensity of the radiation emitted by a single electron is rather low. For the ${ }^{23} \mathrm{Na}$ parameters and statistically independent electrons, a photon is emitted in the mode under consideration each $10^{7}$ electrons. This problem may be overcome by looking at the radiation generated by a very large number of electrons at a time [17]: since the radial velocity $v_{r}$ is much smaller than the charge velocity $w$, the profile of the group cone would not be smeared out even if the spatial extension of the bunch of electrons is much longer than the wavelength of the emitted light.

In summary, we have developed a general theory for Cherenkov emission in arbitrary nonisotropic and dispersive dielectrics and we have given an analytical expression for the group cone over which the intensity of the emitted light is maximum. Unlike in isotropic and nondispersive media, the group cone is here much narrower than the wave cone defined by the usual Cherenkov coherence condition and is orthogonal neither to the phase nor to the group velocity. This conceptual distinction becomes of great physical relevance in media exhibiting slow light propagation. As an illustrative example, we consider the realistic case of a coherently driven ultracold ${ }^{23} \mathrm{Na}$ gas: the geometrical and dispersive properties of the corresponding dielectric ten- sor are shown to allow an experimental characterization of the role of the group velocity in the process of Cherenkov emission.

We thank F. Illuminati, S. de Siena, S. Harris, and G. Afanasiev for useful discussions. We acknowledge support by the INFM (Progetto Ricerca Avanzata "photonmatter") and the European Community (Contract No. HPRICT1999-00111).

*Present address: Laboratoire Kastler-Brossel, Ecole Normale Superieure, 24 rue Lhomond, 75231 Paris Cedex 05, France.

Email address: Iacopo.Carusotto@lkb.ens.fr

[1] M. M. Kash et al., Phys. Rev. Lett. 82, 5229 (1999).

[2] D. Budker, D.F. Kimball, S. M. Rochester, and V. V. Yashchuk, Phys. Rev. Lett. 83, 1767 (1999).

[3] L. V. Hau, S. E. Harris, Z. Dutton, and C. H. Behroozi, Nature (London) 397, 594 (1999).

[4] D. F. Phillips et al., Phys. Rev. Lett. 86, 783 (2001); C. Liu, Z. Dutton, C. H. Behroozi, and L. V. Hau, Nature (London) 409, 490 (2001).

[5] G. S. Agarwal and W. Harshawardhan, Phys. Rev. Lett. 77, 1039 (1996); S. E. Harris and L. V. Hau, ibid. 82, 4611 (1999); H. Schmidt and A. Imamoglu, Opt. Lett. 21, 1936 (1996).

[6] I. M. Frank, Nucl. Instrum. Methods Phys. Res., Sect. A 248, 7 (1986), and references therein.

[7] L.D. Landau and E. M. Lifshitz, Electrodynamics of Continuous Media (Pergamon, London, 1960).

[8] This expression for $\mathbf{v}_{g}$ corresponds to the ratio of the Poynting vector $\mathbf{S}$ and the energy density $u$ [7].

[9] In the electric dipole emission, a similar distinction is done between the near zone $(k r \ll 1)$ and the radiation zone $(k r \gg 1)$; see, e.g., J. D. Jackson, Classical Electrodynamics (Wiley, New York, 1975), 2nd ed.

[10] J. V. Jelley, Cherenkov Radiation and its Applications (Pergamon, London, 1958). See also G. Afanasiev et al., Physica (Amsterdam) 269B, 95 (1999).

[11] In usual EIT experiments the effect of Doppler broadening in hot gases is overcome by choosing a dressing field copropagating with the probe field [12]; unfortunately, the conical geometry of Cherenkov emission does not allow for such a choice, so that one is forced to use an ultracold sample in which Doppler broadening is absent.

[12] E. Arimondo, in Progress in Optics $X X X V$, edited by E. Wolf (Elsevier, New York, 1966), p. 257.

[13] J. F. Cornwell, Group Theory in Physics (Academic, London, 1994).

[14] D. H. Auston, K. P. Cheung, J. A. Valdmanis, and D. A. Kleinman, Phys. Rev. Lett. 53, 1555 (1984).

[15] S. E. Harris and Y. Yamamoto, Phys. Rev. Lett. 81, 3611 (1998).

[16] V. L. Ginsburg and I. M. Frank, Dokl. Akad. Nauk SSSR 56, 699 (1947); L. S. Bogdankevich and B. M. Bolotovskii, Zh. Eksp. Teor. Fiz. 32, 1421 (1957) [Sov. Phys. JETP 5, 1157 (1957)].

[17] J. Ohkuma, S. Okuda, and K. Tsumori, Phys. Rev. Lett. 66, 1967 (1991). 